\title{
LA EDUCACIÓN COMO UN MEDIO PARA ALCANZAR LA PAZ
}

\author{
EDUCATION AS A MEAN TO ACHIEVE PEACE
}

\author{
Juan Carlos Naranjo Segura ${ }^{1}$ \\ jucanase@gmail.com
}

Fecha de recepción: 28 noviembre 2013 - Fecha de aceptación: 9 enero 2014

\begin{abstract}
Resumen
El presente artículo expone algunas de las reflexiones desarrolladas en la investigación titulada "El aporte del programa Ética, estética y ciudadanía al fortalecimiento de los Derechos Humanos de la adolescencia". La cual centra el análisis en la caracterización de la ideología detrás del modelo educativo costarricense, así como en sus consecuencias y la visualización de una educación crítica como un medio para alcanzar la paz.

Palabras claves: Educación, neoliberalismo, capital humano, emancipación y paz.
\end{abstract}

\section{Abstrat}

This paper presents some developed ideas based on the research entitled "The contribution of Ethic programs, aesthetics and citizenship to improve human rights on adolescence." Mainly, the analysis focuses on the characterization of the ideology behind Costa Rican educational model and its consequences as well as the display of a critical education as a way to achieve peace.

Keywords: Education, neoliberalism, human capital, empowerment and peace

\section{Introducción}

Las sociedades estipulan a las escuelas, como las instituciones encargadas de los procesos de socialización de los futuros ciudadanos y ciudadanas, pues mediante esta organización se estructura lo que conocemos como educación formal. Su importancia radica en dos extremos: por una parte, contribuyen a reproducir el orden social y por otra, expresan la esperanza de que pueden mejorar ese dicho orden. Las escuelas caminan orientadas así, entre el pasado y el futuro, y con esto su tarea no es sólo preservar el pasado, sino construir el futuro.

Al comprender la escuela como un medio para la promoción de un proceso educativo en el cual se establecen las bases de una sociedad mejor, que se constituye a partir de la relación entre el conocimiento y la realidad, y sobre la que se le permite actuar; con esto, se aprenden formas de relacionarse con otras personas. Se asimilan ideas sobre uno mismo, sobre nuestro lugar en la estructura social, en la vida y en el mundo, se desarrollan esperanzas y expectativas, y se adquieren habilidades.

1. Profesor del L.E.B. José Figueres Ferrer y de la Escuela de Formación Docente, Sede Rodrigo Facio, Universidad de Costa Rica. 
Así pues, entendiendo lo complejo del proceso educativo y las múltiples variables que lo integran, el presente artículo tiene como objetivo caracterizar el papel del proceso educativo costarricense como un instrumento que favorezca el desarrollo de la paz, convirtiéndose en un instrumento para la transformación social.

No obstante, para poder lograr tal cometido el artículo expone tres partes. En el apartado primero, se busca identificar la ideología económica predominante en el sistema educativo costarricense y la influencia en la formación requerida para el mundo competitivo moderno.

La segunda parte, tiene como meta, examinar las consecuencias en la sociedad del modelo de educación, impulsado a partir de las políticas neoliberales implementadas en los países de América Latina. Finalmente, el tercer apartado busca describir posibles acciones, que partiendo de la pedagogía crítica, se pueden realizar para hacer de la educación un instrumento que favorezca la paz.

\section{Hacia una construcción de la ideología económica dominante en educación}

La década de los setenta como bien lo propone Hobsbawm (1998), es el fin de la década de oro del capitalismo, y la historia de los años veinte que siguió a 1973, es la historia de un mundo que perdió el rumbo y se deslizó hacia la inestabilidad y la crisis.

Las décadas de crisis que siguieron a 1973 no fueron una gran depresión, puesto que la economía global no quebró, aunque la edad de oro finalizó en 1973-1975. Sin embargo, en el mundo capitalista continuó el desarrollo económico.

Por un lado, la crisis de la década de los ochenta, provoca un cuestionamiento en el papel del Estado, tal y como se venía desarrollando décadas atrás. La tesis que emana, expone que el enfoque de producción en el que prevalece la inversión social de los Estados, es un gasto que los países subdesarrollados no pueden mantener. Por esto, bajo esta coyuntura, se explica el germen de las políticas neoliberales como una alternativa a la crisis de la economía.

Por otro lado, con la consolidación del neoliberalismo como la postura política-económica, que sustenta el capitalismo global actual en gran parte del mundo; la educación ha sufrido una serie de transformaciones de fondo y forma muy importantes, las cuales se han llegado a cristalizar, incluso en propuestas curriculares plasmadas en los planes y programas de estudios desde la educación básica hasta el nivel superior. De este modo, en distintos países se han sumado a esta oleada de reformas y transformaciones a partir de los cambios estructurales generados en la década de los ochenta y los noventa, en los ámbitos social y educativo.

El neoliberalismo llevado a la educación, tiene como finalidad aplicar tres conceptos básicos: eficiencia, eficacia y productividad, tal y como se expresa a continuación:

Los neoliberales definen un conjunto de estrategias orientadas a transferir la educación de la esfera de los derechos sociales a la esfera del mercado. Para ellos, la crisis educacional es, antes que nada, una crisis de eficiencia, eficacia y productividad derivada del inevitable efecto perverso al cual conduce la planificación y el centralismo social. (Gentili, 1995: 31)

La ideología que describe la cita anterior implica la comprensión de la forma en que se considera a las instituciones escolares, las cuales deben de asumir dos posiciones: (a) funcionar como pequeñas empresas productoras de servicios educacionales, y (b) asumir los principios de probada eficacia para alcanzar cierto liderazgo en cualquier mercado. Esto implica un proceso de "McDonaldizar"2 la escuela, lo cual supone pensarla como una institución flexible que debe reaccionar a las señales que emite un mercado educacional altamente competitivo.

Además, la educación adquiere un vínculo indisoluble con el modelo de producción sustentado por la economía mundial, en el que se forma para tener una serie de habilidades, destrezas y competencias para adaptarse al mundo competitivo del mercado:

2. El término de McDonaldizar es utilizado por George Ritzer en su libro "La Macdonalización de la sociedad", en donde establece un análisis del impacto del modelo de producción capitalista en nuestra vida cotidiana, partiendo de la descripción de la influencia que ha tenido el modelo de negocio particular de Macdonals, en la sociedad contemporánea. 
En este modelo de organización social, la escuela tiene por función, en la perspectiva de los hombres de negocios, la transmisión de ciertas habilidades y competencias necesarias para que las personas se desempeñen competitivamente en un mercado de trabajo altamente selectivo y cada vez más restringido. La buena educación escolar debe garantizar las funciones de selección, clasificación y jerarquización de los postulantes a los futuros empleos. Para los neoliberales, en esto reside la "función social de la escuela. (Gentili, 1995: 19)

La dinámica que adquiere la escuela bajo esta perspectiva, implica la formación de las personas en función del mercado de trabajo, garantizando la selección del más apto a ocupar el puesto. Entendido de esta forma el proceso educativo, no sólo implica la reducción a la máxima expresión de su papel en medio de la sociedad, sino que visualiza al ser humano como un simple reproductor del sistema, sin capacidad creadora y critica para transformar su entorno.

Siguiendo la visión de Altusser (1988), la escuela es considerada como la mejor forma a través del cual el modelo de producción, es aprendido y reproducido por los seres humanos. Se parte de la consideración de la existencia de un modelo de sociedad considerado como el único que depende de un modo de producción dominante, en el que se definen las distintas relaciones que configuraran el deber ser, dentro del marco de acción de una cultura.

Bajo esta lógica, la educación puede ser entendida como un medio dentro del aparato ideológico del estado (AIE), en el cual los seres humanos reproducen dicha ideología dominante:

Como AlE dominante la escuela constituye el instrumento más acabado de reproducción de las relaciones de producción de tipo capitalista. Para esto atrae a los niños de todas las clases sociales y les inculca durante años de audiencia obligatoria "saberes prácticos" envueltos en la ideología dominante (Saviani, s.f: 8)

La premisa anterior, permite la comprensión del vínculo entre el neoliberalismo y el sistema educativo costarricense; a su vez, esto se visualiza entendiendo cómo el conjunto de ideas que prevalecen en el modelo educativo nacional, están mediatizadas por la percepción de que el estudio es la forma de ser competitivo en el mercado, es decir, la vía para ser exitoso.
Desde la producción capitalista, la educación posee relevancia económica sólo y cuando contribuye a aumentar el rendimiento de los trabajadores. Sin embargo, no es el contenido de la educación lo que determina por sí mismo su carácter productivo, sino la inserción de éste en la producción. De este modo, define así la educación como consumo, si refleja valores tradicionales y la educación como inversión, si responde a una concepción moderna, eficiente y es funcional para el desarrollo:

[...] la inversión en educación es productiva cuando sólo son aquellas habilidades, conocimientos y atributos adquiridos por el hombre y capaces de aumentar la capacidad de trabajo. (De Luca, s.f: 3)

Por un lado, la forma en que los neoliberales definen a la educación, está muy relacionada con el concepto de capital humano tan de moda en los países subdesarrollados, y que describe a la perfección la intencionalidad que se busca alcanzar con la instrucción escolar. En su libro $E l$ capital humano, Sara Finkel plantea la ideología dominante de los organismos educativos nacionales e internacionales y la define como:

El atractivo de la teoría del capital humano para las instituciones capitalistas, tales como el Fondo Monetario Internacional y el Banco Mundial, se encontraba esencialmente en el reconfortante carácter ideológico de su mensaje. La teoría afirmaba que las naciones del Tercer Mundo eran pobres, no a causa de la estructura de las relaciones económicas internacionales, sino debido a características internas, especialmente a su carencia de capital humano. [...] Así se desviaba la atención de las variables estructurales a las individuales (Karabel y Halsey, 1976: 27)

Así, estableció que el gasto en educación no era tal, sino una inversión que aumentaba la capacidad productiva del trabajo, el eje central era concebir a la educación como una inversión, dado que no sólo proporciona beneficios y satisfacciones a los sujetos, sino que incrementa su capacidad y calidad productiva. Para la teoría del capital humano, el individuo, a través del desarrollo de actividades educativas, aumenta aquellas capacidades humanas que mejoran su perspectiva de renta real. De este modo, las personas adquieren capacidades tanto de producción, como de consumo, es decir, como producto de 
una inversión. Así, el proceso educativo favorece la productividad económica.

De acuerdo con esto, la tesis central del capital humano, apuntó que la inversión en educación, desarrolla en la población escolarizada destrezas cognitivas, que a su vez, generan mayor productividad en los centros de empleo. Otra idea básica, se refiere a las decisiones personales de gastar e invertir recursos en educación, con el objetivo de aumentar el flujo de futuros ingresos:

En la actualidad, la mayoría de los proyectos de reforma impuestos o propuestos para los sistemas educativos tienen su anclaje teórico en conceptualizaciones que circulan de manera predominante y constituyen el fundamento racional sobre el que se edifican. Predomina la teoría del capital humano y las ideas derivadas de la concepción de que existe un mercado de la educación (Noriega, 1996: 33)

Asimismo, como ideología, la visión del capital humano que impactó en décadas pasadas, y que aún tiene vigencia, implica poner énfasis en el discurso neoliberal, y en la posibilidad del desarrollo de los pueblos en función (fórmula casi mágica) del incremento de los procesos educativos.

La teoría del capital humano actúa como articulador en la ideología desarrollista en la educación. Esta idea se engancha a la de libertad de elección del sujeto en sí mismo, de modo que, según el capital humano, el proceso educativo proporciona una mayor calificación de la fuerza de trabajo, la cual se refleja en la productividad de los individuos y a su vez, en el incremento de las tasas de crecimiento de la producción, que finalmente impactan a la economía, haciéndola eficiente, y contribuyendo al efectivo desarrollo de las naciones.

No obstante, las premisas básicas del capital humano, bajo el esquema neoliberal, se han endurecido, puesto que se planea dotar de educación a las personas, conforme a las necesidades del sector productivo, y si éste requiere preferentemente personal con habilidades y competencias básicas y en menor escala personal técnico capacitado, pues así se tendrá que diseñar la política educativa, favoreciendo poco a poco, a través de mecanismos eficientes, la paulatina mercantilización del servicio educativo, sobre todo para el nivel superior.
Por otro lado, comprendiendo lo anterior, es que se pone en boga el llamado según Moreno (2002), "modelo educativo por competencias", para Laval (2004) "Pedagogía de las competencias", Argüelles (2002) refiere como "educación basada en competencias" entre otras denominaciones que recibe, las cuales son ya una realidad en las propuestas y desarrollo curricular en los centros escolares de todos los niveles educativos en nuestro país, y en varios países de América Latina y el Caribe.

Además, el auge de la teoría del modelo de competencias, se ha consolidado como la teoría pedagógica de las políticas educativas neoliberales en el mundo; hecho que sin duda ha sido propiciado por el gran impulso que le han brindado en los últimos años organismos internacionales, tales como: el Banco Mundial (BM), la Organización para la Cooperación y Desarrollo Económico (OCDE), y la Organización de las Naciones Unidas para la Educación, la Ciencia y la Cultura (UNESCO), aunque con diferentes énfasis, ésta última.

Así pues, el modelo de educación por competencias, se utiliza a partir de palabras claves como "habilidades, destrezas y conocimientos", y es concebido como un conocimiento ligado al saber hacer. La competencia:

“[...] no se limita a los aspectos procedimentales del conocimiento, a la mera posesión de habilidades y destrezas, sino que se ve acompañada necesariamente de elementos teóricos y actitudinales" (Díaz y Rigo, 2002: 79)

Los aspectos anteriores parecen filtrarse con mayor facilidad en los niveles básicos, puesto que las competencias se integran a los saberes conceptuales y actitudinales, con cierta naturalidad por los esquemas curriculares de formación de los educandos. No obstante, en los niveles superiores, sobre todo en los ámbitos de la educación tecnológica, parece imponerse una dimensión pragmática de la educación para el sector productivo, en el que la capacitación, más que formación en competencias laborales específicas y/o profesionales, se imponen en el anclaje del vínculo escuela-empresa, dejando de lado la función social originaria integral de esas instituciones. 
Sin embargo, se debe comprender los efectos de dicho discurso, esto en cuanto tiende a culpabilizar y visualizar al fracaso como el resultado de las condiciones individuales, y no el fruto de la estructura desigual de la sociedad, como se mostrará a continuación.

\section{Más allá del discurso ideológico económico del Neoliberalismo: el descalabro de un modelo de educación}

Este apartado tiene como objetivo, examinar el impacto del modelo de educación impulsado a partir de las políticas neoliberales implementadas en los países de América Latina, sobre todo en el caso costarricense. Se trata de decodificar la teoría de capital humano y la pedagogía por competencias, para comprender su impacto real sobre la sociedad costarricense.

En relación con esto, el elemento discursivo que está presente en la teoría del capital humano y por competencias, es la idea que fundamenta la educación como un instrumento esencial para el crecimiento y desarrollo de la economía, tal y como sucede en países altamente desarrollados.

Por su parte, el punto que se va a profundizar implica la reflexión acerca de cómo ese modelo de educación, al implantarse en países como el nuestro, forma parte de la falacia o espejismo ideológico que se adhiere a un discurso; lejos de llevarse a cabo en la realidad, sirve para disfrazar las verdaderas fuentes estructurales de la pobreza y el subdesarrollo de los pueblos, circunstancias que bajo los parámetros del neoliberalismo económico, se han agudizado a niveles preocupantes incluso para las propias agencias del capital mundial.

De esta manera, la ideología del neoliberalismo inmersa en el sistema escolar es abominable, pues permite naturalizar las desigualdades producidas por el mercado, a un nivel macro. Lo anterior implica la reproducción de las desigualdades a través del proceso educativo, las cuales no son cuestionadas, sino consideradas como la consecuencia de la escasez de habilidades, destrezas y competencias que se posee a nivel individual, como se muestra a continuación:
La ideología presente en la cultura escolar enseña a los estudiantes a ver el fracaso como algo individual que resulta de su escasez de habilidad, desarrollando unos principios que priorizan las diferencias y divisiones en términos de rendimiento académico; consecuentemente, el sistema de enseñanza se encarga de transformar las diferencias y desigualdades extraescolares en desigualdades reales de aprendizaje o de capital cultural. De esa manera la igualdad formal, que rige los principios y las prácticas escolares, ha servido para enmascarar, más que para superar, las desigualdades reales ante la enseñanza y la cultura enseñada y exigida. (Pérez, 2000:196)

Así pues, la consecuencia más visible es la naturalización de las desigualdades, en un discurso en el cual el sistema pareciera ser perfecto, al manifestar que es el mercado el único ordenamiento económico racional, y al definir las relaciones sociales y la forma en que los individuos adquieran lo necesario para su vida.

De acuerdo con estas ideas, la consecuencia de la educación como reproductora del orden dominante, implica desenmascarar la formación escolar como el sustento en que se desarrolla la violencia simbólica de la sociedad moderna, la cual se establece cuando la desigualdad es legitimada a partir del proceso de dominación de las clases poderosas sobre los grupos subalternos, tal y como se muestra a continuación:

Se ve, entonces, que el reforzamiento de la violencia material se da por su conversión en el plano simbólico donde se produce y reproduce el reconocimiento de la dominación y de su legitimidad por el desconocimiento (disimulación) de su carácter de violencia explícita. Así, a la violencia material (dominación económica) ejercida por los grupos o clases dominantes sobre los grupos o clases dominadas corresponde la violencia simbólica (dominación cultural). (Saviani, s.f: 8)

Por tanto, la violencia simbólica implica decodificar la forma en que se mantiene el orden social establecido, es decir, el desconocimiento de la mayoría de los explotados de la causa de su realidad: la dominación económica como la causante de las enormes desigualdades prevalecientes en la sociedad moderna. Sin embargo, la institucionalidad educativa cumple un papel socializador de este proceso.

Sin lugar a dudas, esta es la función necesaria de la educación dentro del sistema, pues es la forma a través de la cual disimula, y por eso cumple eficazmente, su función de marginación. 
Es así como se configura un sistema educativo incapaz de crear condiciones que favorezcan el desarrollo de una sociedad en la cual se fundamenten los valores de igualdad, justicia y paz. A su vez, el sistema condensa a lo interno las mismas condiciones que reflejan estas desigualdades a nivel macro en la sociedad; la mayor expresión de lo anterior es la problemática de la expulsión escolar, tal y como se expresa a continuación:

Solo dos de cada diez estudiantes terminan la secundaria sin repetir ningún curso. La consecuencia es obvia para los estudiantes, pero también para el fisco: cada graduado nos cuesta casi diez años de colegio, en vez de cinco. Además, sabemos que la probabilidad de conseguir un buen trabajo está ahí solo para quienes tienen algo más que secundaria: la secundaria es apenas un piso. Sin embargo, solo una tercera parte de quienes entran a primer grado se gradúan de secundaria: ¡mantenemos bajo el piso a dos terceras partes de nuestras y nuestros jóvenes! Tal es el reto que enfrentamos desde el MEP, un reto que tiene que ver tanto con la cobertura como con la calidad y la relevancia de nuestra educación. ¿Qué hacer? (Garnier, 2009)

El panorama que describe la cita anterior, permite comprender la forma en que el sistema se reproduce en la institucionalidad educativa, recreando la principal problemática de la sociedad costarricense: la desigualdad. Para el Ministro Leonardo Garnier, está claro que la explicación a tal fenómeno tiene que ver con la calidad y la relevancia de la educación, elementos que forman parte del discurso neoliberal.

Esta realidad también se describe en el Sexto Informe sobre el Estado de los Derechos de la Niñez y Adolescencia en Costa Rica (2008), el que se puntualizan cuatro retos presentes en el sistema educativo nacional, a saber: la cobertura del sistema educativo, la rezaga, repitencia y expulsión, la calidad de la educación y la relación educación pública y privada.

El balance descrito en los anteriores cuatro desafíos, tiene como característica principal, la estrecha relación presente entre ellos. Por un lado, se encuentra el reto de la cobertura de la educación secundaria, en el cual, según dicho informe, se presenta una abrupta disminución entre el casi $100 \%$ de la cobertura de la población en educación primaria, a un $60 \%$ en secundaria, lo cual se podría interpretar como un modelo de educación que es excluyente, en cuanto expulsa a quienes no son capaces de adaptarse a él.

De esta forma el Informe del 2008 (UNICEFUCR), contabiliza que para el 2006 la población expulsada ${ }^{3}$ corresponde al 15,2\% de hombres y de $11,5 \%$ de mujeres, lo cual enmarca una cruda realidad, pues manifiesta una sociedad que se va construyendo cada vez más a partir de la desigualdad, tal y como se expresa en la siguiente cita:

De esta forma, la expulsión del sistema educativo se revela como un hecho que refleja tanto desigualdades estructurales de la sociedad costarricense, como deficiencias profundas en el sistema educativo, ente que se ha mostrado incapaz de responder a las necesidades de aprendizaje y acceso a la educación de este sector de la población (UNICEF- UCR, 2008: 73)

Unido a lo anterior, se establece el fenómeno de la extra edad, es decir, la población de personas menores de edad que cursan los cursos lectivos en edades superiores a las estipuladas para el desarrollo del plan de estudio, tal y como se presenta a continuación: "se trata de estudiantes de Primer Ciclo, mayores de nueve años, estudiantes de Segundo Ciclo, mayores de doce años y repitentes" (UNICEF- UCR, 2002).

Ejemplo de lo anterior, son los y las estudiantes que logran graduarse en la secundaria en un tiempo de nueve años para poder obtener ese título. Por lo tanto, la repitencia se convierte en un reto, puesto que es la manifestación de un sistema poco atractivo a los intereses del estudiantado, tal y como lo muestra la Primera Encuesta Nacional de Juventud, la cual describe que en los hombres

3 Se habla de expulsión cuando en los centros educativos del Estado, se desarrollan una serie de condiciones ligados a temas económicos, sociales, laborales, que obligan a las personas menores de edad a que abandonen el sistema educativo, por eso cuando se habla de expulsión, se relaciona con abandonar, con dejar, excluir, rechazar, con lo cual adquiere una connotación distinta al termino deserción escolar, el cual es un concepto que se utiliza para referirse a aquellos alumnos que dejan de asistir a clase y quedan fuera.

La expulsión implica el reconocimiento de que dentro del mismo sistema existen condiciones que llevan para que se desarrolle el abandono, en donde los y las estudiantes son las víctimas y el Estado el responsable, mientras la categoría de deserción, se pone la responsabilidad al estudiante en cuanto es quien abandona las aulas. 
entre 15 y 17 años, que no estudian, la principal razón para no hacerlo es el desinterés; lo mismo pasa con las mujeres de este grupo, correspondiendo al 6,6\%. En estos casos, es más atractivo el mercado laboral, que un modelo de educación que no les muestra nada para su precaria situación social, lo que conlleva al fenómeno del trabajo infantil.

En síntesis, queda argumentado tal y como lo expresa el Semanario Universidad (2011), que la formación en las escuelas y colegios del país al ser impartida por igual, sin tomar en cuenta las características y necesidades de cada sector socioeconómico, genera grandes brechas. ${ }^{4}$

Por otra parte, a pesar de las políticas que se han implementado para democratizar el acceso a la educación, y al existir programas y metodologías iguales ciertos sectores resultan afectados. Pues la poca calidad de los programas, el desinterés de los estudiantes y profesorado y la expulsión, son las consecuencias de un sistema que promueve la inequidad.

Paradójicamente a lo expuesto por la ideología neoliberal, el contexto desarrollado por este modelo de educación repercute en el mercado laboral, porque el grado académico constituye una base para obtener trabajos de cierta calidad, pues las personas que no obtienen el título se exponen a empleos mal pagados o informales. De esta manera, la educación cumple su papel de selección de la mano de obra más apta para ciertos oficios o profesiones, definiendo la división social del trabajo en el que se sustente el orden económico mundial y las desigualdades sociales.

La consecuencia de todo lo anterior se describe en la disyuntiva entre el discurso y la realidad: el discurso dominante, plantea la necesidad de una educación de calidad que favorezca la posibilidad del cambio social ascendente en estudiantes de escasos recursos económicos; mientras la realidad de la educación con un tinte neoliberal promueve el abandono del papel social

4 El artículo del Semanario Universidad, hace un comentario a la investigación titulada: "Desigualdad y Pobreza en Costa Rica", del Centro de Estudios Democráticos de América Latina. Una investigación en donde expone como el sistema educativo propicia la construcción de una sociedad más desigual. como agente de movilidad social, evidenciado en la forma en que está estructurado el sistema y la sociedad como tal, conformando un todo que reproduce la desigualdad, tal y como se muestra en los siguientes datos:

"Uno de los obstáculos, citó, es que las 56 universidades gradúan 35.000 profesionales al año, y el mercado laboral solo ofrece 17.000 empleos, lo que indica que la educación ha perdido la posibilidad de garantizar movilidad social" (Rojas, 2011: 4)

Así, lo expuesto con anterioridad, explica lo que teóricos como Laval $(2004)^{5}$ han denominado: "la desintegración del funcionamiento de la escuela", el cual se genera como consecuencia de la introducción de los mecanismos de mercado al ámbito escolar, debido a que el nuevo modelo comercial de escuela funciona con base en la diversidad y diferenciación, a partir de las demandas del público usuario.

De esta forma, la realidad educativa contextualizada en la corriente neoliberal, constituye un marco de acción que se convierte cada vez más en un ente que propicia lo que Galtung (1993), denomina "violencia estructural", principal causa de la inexistencia de la paz.

$5 \quad$ El autor expone que la institución escolar está sufriendo una mutación que se puede asociar con tres tendencias principales: la desinstitucionalización, desvalorización y desintegración. La primera tendencia tiene que ver con que la escuela se empieza a concebir como instancia suministradora de servicios de organización flexible, que aspira a un modelo de empresa educadora, administrada según los principios de la gestión empresarial, bajo presiones que le obligarán a brindar resultados e innovaciones concretas.

En cuanto a la tendencia de la desvalorización, Laval afirma: "Los objetivos que se pueden llamar "clásicos" de emancipación política y de realización personal que se habían asignado a la institución escolar, se sustituyen por los imperativos prioritarios de la eficacia productiva y de la inserción laboral" (Laval, 2004; 26).

El autor indica que aun cuando en los discursos oficiales a nivel mundial, se reconoce a la educación como factor social indispensable para el progreso, sus finalidades y fundamentos culturales y sociales, han sido erosionados, ya que se está dando una transmutación progresiva de sus valores hacia el valor prioritario del neoliberalismo: el económico. Así, Laval muestra su preocupación por que se soslaye la función social de la educación en aras de los intereses económicos de los capitalistas. 


\section{Emancipación: la alternativa para hacer de la educación un instrumento para construir la paz}

Este apartado trasciende la manera en que se ha comprendido a la educación como simple reproductora del sistema económico imperante, de los apartados anteriores. Se parte de una visión de educación, en el que el acceso al conocimiento se convierte en una forma de resistencia a la cultura dominante.

Una forma que visualiza a la educación como un medio de transformación de la sociedad, que permite el conocimiento de la realidad política, económica y social, para transformarla cuando sea necesario, se expresa con De Luca:

Entonces, ante el peligro de que la educación se convierta en una forma de ganarse la vida y deje de ser una experiencia que permita el derecho al conocimiento, necesidad básica del ser humano, los docentes junto a los padres deben sentarse a construir una escuela que sirva como espacio de resistencia de la cultura dominante, no se trata sólo de aprender a vivir en sociedad sino a cambiarla cuando sea necesario, no se puede responder a un niño: no pierdas tiempo aprendiendo estas cosas, no son rentables. (De Luca, s,f: 12)

Por lo tanto, la comprensión del proceso educativo más allá del nexo con la ideología económica dominante, permite recuperar el papel de la educación como un instrumento que pueda permitir hacer rupturas en el orden social dominante. De ahí que para lograr trascender la visión de Althusser sobre la educación como un simple reproductor de la ideología del Estado, se asume el concepto de educación problematizadora de Freire.

Esta posición teórica de educación, no entiende el proceso educativo como un mero depósito de conocimientos, sino que es un acto cognoscente que sirve a la liberación, en cuanto permite la comprensión crítica de la realidad, tal y como se expresa a continuación:

“[...] Mi visión de la alfabetización va más allá del ba, be, bi, bo, bu. Porque implica una comprensión crítica de la realidad social, política y económica en la que está el alfabetizado" (Freire, 1997: 83)

No obstante, para poder dar un sustento teórico a esta forma de entender a la educación, se asume como marco de referencia a la pedagogía crítica, puesto que emerge como un insumo para convertir al proceso educativo en una herramienta para la comprensión crítica de la realidad, pues parte de la profunda insatisfacción que genera una sociedad injusta y de la voluntad de transformarla.

En relación con esto, Ramón Flecha destaca la participación social, la transformación del contexto y el diálogo como las claves de una educación crítica (Flecha, 1997: 13-46):

- Suele hablarse en cualquier modelo educativo no transmisivo, pero no necesariamente crítico, de la participación como principio pedagógico. En algunos casos, esto ha llegado a formularse de manera más radical, partiendo de la necesidad y el derecho del alumnado a la negociación del currículo. Partiendo de ello, Juan Bautista Martínez Rodríguez ha propuesto que la negociación del currículo con el alumnado se convierta en una realidad, pero teniendo en cuenta la desigualdad de poder de ciertos grupos sociales. Por ejemplo, al negociar los contenidos: es necesario reconocer que existen dos culturas en interacción, la popular y la dominante "quien ve la cultura legítima desde el punto de vista de la cultura dominada no ve la misma cultura que aquel que se ve [sic] legitimada su posición por la misma cultura" (Martínez, 1999: 160)

- La transformación del contexto, en la educación emancipatoria, "el conocimiento no se estudia por sí mismo, sino que es contemplado como una mediación entre el individuo y la realidad social más amplia" (Giroux, 1990: 110). El conocimiento es también de otro tipo: a diferencia del conocimiento técnico-instrumental (causa-consecuencia) o del práctico (entendimiento, interpretación), el conocimiento crítico se corresponde con un interés emancipador: tomar el control de las propias vidas y transformar la realidad, mediante la reflexión y control sobre las metas y no sólo sobre los medios. Incluye así a los otros tipos de conocimiento al abarcarlos en la praxis desde una perspectiva 
autorreflexiva, la praxis realiza la síntesis entre teoría y práctica, es la acción consciente y reflexiva que desborda los límites de lo concreto, tanto acción como reflexión, dado que abarca lo que es externo al acto concreto, para ir más allá de él.

- La relación entre acción-práctica y teoríareflexión es dialéctica, pues si el pensamiento influye en la acción, ésta también transforma el pensamiento, al ponerse éste en contacto con lo concreto. Freire define la concienciación, como el "proceso mediante el cual los seres humanos participan críticamente en un acto transformador" (Freire, 1990: 120), y defiende la necesidad tanto del contexto teórico (cuando se produce la reflexión) como de la inserción en el contexto concreto, la realidad social que ha de ser transformada:

- El diálogo: La educación emancipadora, ya no ha de entenderse como una acción de unas personas sobre otras, pues sería inconsecuente con sus presupuestos básicos: "nadie educa a nadie - nadie se educa a sí mismo los hombres se educan entre sí mediatizados por el mundo" (Freire, 1974: 73)

Es evidente, que la pedagogía crítica enfatiza la importancia al argumento de que el conocimiento adquirido debe ser puesto en práctica, retomando el carácter eminentemente político que posee la educación. Según Peter McLaren (1997), la pedagogía crítica invita a analizar la relación entre experiencia, conocimiento y orden social, con una perspectiva transformadora:

Todo el proyecto de la pedagogía crítica está dirigido a invitar a los estudiantes y a los profesores a analizar la relación entre sus propias experiencias cotidianas, sus prácticas pedagógicas de aula, los conocimientos que producen, y las disposiciones sociales, culturales y económicas del orden social en general (...). La pedagogía crítica se ocupa de ayudar a los estudiantes a cuestionar la formación de sus subjetividades en el contexto de las avanzadas formaciones capitalistas con la intención de generar prácticas pedagógicas que sean no racistas, no sexistas, no homofóbicas y que estén dirigidas hacia la transformación del orden social general en interés de una mayor justicia racial, de género y económica (Mc Laren, 1997: 270)
Asimismo, la educación, como práctica de la libertad, implica la negación del hombre aislado del mundo, propiciando la integración, y la construcción del conocimiento se dará en función de la reflexión que no deberá ser una mera abstracción. Por esto el hombre, siempre deberá ser comprendido en relación con su vínculo con el mundo.

De este modo, se parte de la noción de una pedagogía de la liberación, comprendiendo que no hay educación liberadora si no pensamos que hay algo de qué liberarse, y no hay educación transformadora si no se siente un deseo y una posibilidad de cambio social. Sin embargo, no es necesario estar de acuerdo en un mismo modelo ideal, ni siquiera tener una alternativa global ya diseñada, sino compartir una orientación utópica para superar las limitaciones del presente, y creer que la educación no puede ni debe rehuir sus responsabilidades.

Partiendo de estas ideas, el proceso educativo se convierte en una forma de emancipación del grupo de estudiantes, es decir, la educación permite el desarrollo del empoderamiento. Lo anterior se alcanza mediante la formación que propicia el desarrollo de la autonomía, permitiendo que los sujetos asuman su propio proyecto de vida en medio de su contexto cultural, el cual es entendido y aceptado, pero también transformado en la medida que no respeta su dignidad humana. Por tanto, entendida de esta manera, la educación debe de ser un medio para el logro de una sociedad más justa, y por ende, que promueva el desarrollo de paz.

Es necesario entonces, que el proceso de educación permita la comprensión de la realidad social que es vivida por los sujetos que aprenden, para ello es necesario no sólo la memorización de contenidos, sino desarrollar en el grupo de estudiantes, la capacidad de la crítica, en el que a partir de la acción y reflexión se logra la emancipación, tal y como se muestra en el siguiente texto:

Así el interés emancipador constituye un esfuerzo para que los grupos de personas se comprometan en una acción autónoma. Esto se deriva del desarrollo de ideas críticas, auténticas respecto a la base de la construcción de la sociedad humana por los miembros de tales grupos (Grundy, 1991:160) 
Finalmente y dadas estas condiciones, se puede visualizar a la educación como un medio para alcanzar la paz (entendiendo paz como la ausencia de violencia). En este caso, una educación que propicie la emancipación, implica establecer acciones dentro del modelo educativo que promuevan el cuestionamiento del orden social establecido. Solamente de esta forma, la educación puede dar un aporte trascendental a procesos reales de paz.

\section{A modo de conclusión}

Los primeros apartados del presenten trabajo, reflejan parte de la realidad que explica el sistema educativo costarricense. Con ellos se buscó establecer una contextualización que permita la comprensión del vínculo existente entre la ideología neoliberal, bajo la acepción de formación para el mercado laboral, y las consecuencias en una sociedad cada vez más desigual.

Al reconstruir la ideología detrás de la educación y sus consecuencias, se logra evidenciar cómo el contexto societario está configurado para la inexistencia de procesos auténticos de paz. De ahí que se utilice incluso el concepto de violencia simbólicacomo la forma de explicar cómo culturalmente se aceptan las situaciones de una estructura que atentan contra el desarrollo de la gran mayoría de personas.

Es en este punto, que aparece un modelo de educación capaz de cuestionar dicho orden, considerado como "normal", y propiciar procesos en los que el acceso al conocimiento de la realidad, que se permite crear condiciones para el cambio.

Así como lo expuso Henry Giroux, en la conferencia que dio en el Congreso Nacional organizado por la Asociación de Diarios de la República Argentina:

[...] lo que se analiza aquí es la necesidad de educar a los alumnos con el conocimiento y habilidades que necesitarán para participar en el mundo público, para ser actores de un gran escenario y participar en la conversación pública que prevalece acerca de temas educativos, políticos, sociales y culturales. Esto sugiere prácticas educativas que conecten el pensamiento crítico con la acción colectiva, el conocimiento y poder con una impaciencia profunda con respecto al status quo, y a la mediación humana para la responsabilidad social (Giroux, 2000)
Por consiguiente, no se trata de una metodología, ni mucho menos un recetario que permite hacer de la educación el medio para llegar a la paz. Tampoco se trata de darle a la educación un papel mesiánico para redimir los males de un modelo de producción atroz como lo es el capitalista. Se trata de comprender la educación como un instrumento de poder, en cuanto permite que los y las estudiantes asuman su papel como actores sociales, el cual el conocimiento crítico de la realidad, permita el desarrollo de procesos de organización social, de movilidad, que originen rupturas en la forma en que se estructura la cultura.

En fin, hablar de paz, es hablar de la lucha que se debe hacer para alcanzarla. Es decir, la paz se construye, se lucha y se alcanza mediante esfuerzos colectivos, en la cual, la responsabilidad individual inicia a través de procesos de decodificación del entorno que los rodea. La educación se vuelve un instrumento de poder, en la medida que debe de propiciar el cuestionamiento de la propia realidad, la cultura y la sociedad que lo envuelve. Por lo tanto, para alcanzar la paz, es necesario de un proceso de educación que permita el desarrollo de la capacidad crítica y creadora del ser humano.

\section{Referencias bibliográficas}

Althusser, (1988). Ideología y aparatos ideológicos del Estado. (trad.). Freud y Lacan. Buenos Aires: Nueva Visión.

De Luca, L.S. (s.f). La Escuela como agente socializador. ¿Enseñar para adaptarse a la sociedad o para transformarla? análisis de posturas y presupuestos teóricos y metodológicos. [Artículo en línea de la Revista Iberoamericana de Educación]. Recuperado el 21 de marzo del 2012, disponible en: www.rieoei.org/ deloslectores/406DeLuca.pdf.

Díaz, F y Rigo, M. (2002). Formación docente y educación basada en competencias. En: Valle Flores, María de los Ángeles. (coord.) Formación en competencias y certificación profesional). México: CESU_ UNAM. 
Finkel, S.(s.f). El "Capital Humano": concepto ideológico. Documento "Santa Fe" una estrategia para América Latina en los años 90.

Flecha, R. (1997). "Pensamiento y acción crítica en la sociedad de la información". En Goikoetxea, J. y García (1997). Ensayos de pedagogía crítica. España: Popular

Freire, Paulo (1974). Concientización. Buenos Aires: Búsqueda.

Freire, P. (1990). La naturaleza política de la educación. Barcelona: Paidós.

Freire, P (1997). Pedagogía del Oprimido. Recuperado el 24 de febrero del 2010, disponible en: www.servicioskoinonia.org/.../ FreirePedagogiadelOprimido.pdf

Freire, P. (1997). Pedagogía da autonomía. Saberes necessários à prática educativa. Rio de Janeiro: Paz e Terra.

Galtung, J. (1993), Paz. En Rubio, A. Presupuestos teóricos y éticos sobre la paz. España: Universidad de Granada.

Garnier, L. 2009. "Un nuevo estilo en Educación". La Nación. Domingo 27 de agosto, 2006. Recuperado el 21 de octubre del 2011, disponible en: http://www.nacion. com/ln_ee/2006/agosto/27/opinion6.html.

Gentili,. (1995) Cultura, Política y Currículum. Ensayo sobre las crisis de la Escuela Pública. Buenos Aires: Losada.

Giroux, H.A. (1990). Los profesores como intelectuales. Barcelona: Paidós.

Grundy, S (1991). Producto o praxis del Currículum. Madrid: Morata.

Hobsbawm, E. (1998). Historia del siglo XX. Buenos Aires: CRÍTICA (Grijalbo Mondadori, S.A.)

Karabel J. y A. H. Halsey (1976). La investigación educativa: una revisión e interpretación. (Trad.) Jorg G. Vatalas, M. Teobaldo y S. Llomotave. Nueva York: Oxford University Press

Laval, Christian. (2004). La escuela no es una empresa. El ataque neoliberal a la enseñanza pública. España: Paidós.

Martínez, J. (1999). Negociación del currículum. La relación enseñanza-aprendizaje en el trabajo escolar. Madrid: La Muralla.
McLaren, P. (1997). Pedagogía crítica y cultura depredadora. Barcelona: Paidós.

Noriega, M. (1996). En los laberintos de la modernidad: Globalización y sistemas educativos. México: Universidad Pedagógica Nacional.

Pérez, C (2000). La escuela frente a las desigualdades sociales. Apuntes sociológicos sobre el pensamiento docente. Revista Iberoamericana de Educación. $\mathrm{n}^{\circ} 23$. [Revista en Línea]. Recuperado el 8 de mayo del 2011, disponible en: http://www. rieoei.org/index.php.

Ponencia presentada en el Foro COPLPROCE: "La doctrina del neoliberalismo y el proceso de globalización en los países subdesarrollados", por Rafael Pleitez, jefe del Departamento de Economía de la Universidad Centroamericana "José Simeón Cañas" (UCA), 12 de agosto de 1998.

Rojas, N. (2011). Focos de desarrollo se concentran en Gran Área Metropolitana: Sistema educativo costarricense propicia desigualdad. Semanario Universidad. Del 29 de junio al 5 de julio de 2011. $\mathrm{N}^{\circ} 104$, San José, Costa Rica.

Saviani, D. (s.f). Las teorías de la educación y el problema de la marginalidad en América Latina. Traducción de Susana Vior, Publicado en la Revista Argentina de Educación y en Diálogos. [Revista en línea]. Recuperado el 5 de setiembre del 2011, disponible en: www.pedagogica.edu.co/ storage/rce/articulos/13_04ens.pd.

Torres, C A. (2008). Después de la tormenta neoliberal: la política educativa latinoamericana entre la crítica y la utopía. Revista Iberoamericana de Educación. N. 48 pp. 207-229. Recuperado el 23 de junio del 2011, disponible en: http://www.rieoei.org/ index.php.

UCR y UNICEF. (2008) Sexto Informe sobre el Estado de los Derechos de la Niñez y Adolescencia en Costa Rica. San José, Costa Rica: Universidad de Costa Rica. 
